\title{
Carta de Porto Alegre sobre Saúde Planetária (10 Simpósio Internacional de Saúde Planetária, Porto Alegre, 2017)
}

\section{Porto Alegre Charter on Planetary Health \\ ( $1^{\text {st }}$ International Symposium on Planetary Health, Porto Alegre, 2017)}

\section{Carta de Porto Alegre sobre Salud Planetaria}

\section{(1ํ Simposio Internacional de Salud Planetaria, Porto Alegre, 2017)}

\author{
Sergio Antonio Sirena ${ }^{1,2} \odot$, Julio Baldisserotto ${ }^{1,3}$, Airton Tetelbon Stein ${ }^{1,4}$, Carlos Dora ${ }^{5}$, Enrique Barros ${ }^{1}$, Geraldo Pereira Jotz ${ }^{1,3}$ \\ ${ }^{1}$ Grupo Hospitalar Conceição (GHC). Porto Alegre, RS, Brasil. \\ ${ }^{2}$ Universidade de Caxias do Sul (UCS). Porto Alegre, RS, Brasil. \\ ${ }^{3}$ Universidade Federal do Rio Grande do Sul (UFRGS). Porto Alegre, RS, Brasil. \\ ${ }^{4}$ Universidade Federal de Ciências da Saúde de Porto Alegre (UFCSPA). Porto Alegre, RS, Brasil. \\ ${ }^{5}$ Columbia University Mailman School of Public Health. New York, NY, Estados Unidos.
}

\section{Resumo}

Os participantes do 1 Simpósio Internacional de Saúde Planetária, reunidos em Porto Alegre, propõem que se dissemine a discussão do tema e se articule a resposta e a contribuição do sistema de saúde aos crescentes desafios que enfrenta a saúde da população decorrentes da mudança climática, poluição, redução da biodiversidade e outros fatores ambientais.

Palavras-chave: Mudança Climática; Biodiversidade; Poluição Ambiental; Sistemas de Saúde

\begin{abstract}
The participants of the $1^{\text {st }}$ International Symposium on Planetary Health, held in Porto Alegre, propose to disseminate the discussion of the theme and articulate the response and contribution of the health system to the growing challenges facing the health of the population due to climate change, pollution, reduction of biodiversity and other environmental factors.
\end{abstract}

Keywords: Climate Change; Biodiversity; Environmental Pollution; Health Systems

\section{Resumen}

Los participantes del 1ㅇ Simposio Internacional de Salud Planetaria, reunidos en Porto Alegre, proponen la diseminación de la discusión del tema y articulación de la respuesta y la contribución del sistema de salud a los crecientes desafíos que enfrenta la salud de la población derivada del cambio climático, contaminación, reducción de la biodiversidad y otros factores ambientales.

Palabras clave: Cambio Climático; Biodiversidad; Contaminación Ambiental; Sistemas de Salud

Como citar: Sirena SA, Baldisserotto J, Stein AT, Dora C, Barros E, Jotz GP. Carta de Porto Alegre sobre Saúde Planetária (1 Simpósio Internacional de Saúde Planetária, Porto Alegre, 2017). Rev Bras Med Fam Comunidade. 2019;14(41):1899. https://doi.org/10.5712/rbmfc14(41)1899

Autor correspondente: Sergio Antonio Sirena. E-mail: sergiosirena@hotmail.com Fonte de financiamento: declaram não haver. Parecer CEP: não se aplica. Procedência e revisão por pares: revisado por pares. Recebido em: 15/10/2018. Aprovado em: 17/03/2019. 
"As mais importantes e atuais ameaças à humanidade são consequência da capacidade intelectual humana; nossa única esperança para superá-las está nessa aptidão”

A Saúde Planetária² diz respeito às interdependências entre saúde da civilização humana e o estado dos sistemas naturais. Na primeira dimensão, estuda a saúde humana dentro do marco dos determinantes sociais, tendo saúde, equidade e justiça social como norteadores. Concretamente, inclui doenças crônicas e infecciosas, mudanças climáticas, acidificação dos oceanos, poluição química, entre outros. Em segundo lugar, estuda as conexões com sistemas naturais nos quais vivemos, a saúde e a diversidade da biosfera. Parte do entendimento de que vivemos em um espaço operacional global seguro e que, se seus limites forem violados, as condições para a nossa sobrevivência serão afetadas.

Os ecossistemas são bases reconhecidas para os Objetivos de Desenvolvimento Sustentável da Organização das Nações Unidas (ONU). ${ }^{3}$ Entretanto, estão sendo degradados aceleradamente pela ação do homem, alguns deles sem precedentes na história, dando surgimento ao Antropoceno, que está por entrar na Escala de Tempo Geológico, com efeitos documentados e estimados e outros ainda não conhecidos e não quantificados sobre a saúde humana.

Os participantes do 1 Simpósio Internacional de Saúde Planetária, reunidos em Porto Alegre, propõem que se dissemine a discussão do tema e se articule a resposta e a contribuição do sistema de saúde aos crescentes desafios que enfrenta a saúde da população decorrentes da mudança climática, poluição, redução da biodiversidade e outros fatores ambientais.

Dentre as propostas e reflexões levantadas, podemos destacar:

- Elaboração de uma agenda de ação em Saúde Planetária a partir da Atenção Primária à Saúde (APS). Desenvolvendo ações para a construção de um modelo de resposta local aos desafios da saúde planetária; uma agenda necessariamente intersetorial que inclua a capacitação de profissionais da saúde para potencializar suas habilidades na orientação dos cidadãos e comunidades para a redução de riscos ao meio ambiente, para a saúde e avaliações de impactos ambientais e sociais.

- O conceito de Saúde Planetária deve ser disseminado e incluído no currículo básico das universidades, escolas técnicas, escolas de ensino fundamental e médio e de aperfeiçoamento profissional.

- Integração da sustentabilidade em nossos comportamentos individuais e coletivos, na prática profissional e no desenvolvimento de políticas públicas.

- Promoção e geração de novos conhecimentos em linhas de pesquisa que possam fundamentar as ações com base em evidências científicas.

- Inserção do tema Saúde Planetária nos currículos de formação dos profissionais da área da saúde, em especial no rol de competências do Médico de Família e Comunidade. 
- Priorização de ações de cunho coletivo e equitativo para um futuro saudável, reconhecendo que poluição do ar é um dos principais fatores de risco de morbimortalidade.

- Inclusão das populações rurais e urbanas marginalizadas nas possíveis atividades dos agentes comunitários para ações relacionadas à Saúde Planetária.

- A Atenção Primária à Saúde (APS) tem um papel fundamental na transformação social necessária para obter desenvolvimento humano dentro do potencial e dos limites que oferece o nosso planeta. É cenário propício para o desenvolvimento de ações que redundem na facilitação de mudanças de comportamento e engajamento das comunidades e sociedade civil na adoção de estilo de vida compatível e apoio às políticas públicas e modelos de desenvolvimento econômico que conduzam à saúde planetária.

- Orientar e capacitar populações que vivem em áreas de risco para desastres naturais buscando a redução da vulnerabilidade e aumento da resiliência.

- Promover a capacitação dos mais diversos profissionais para lidar com catástrofes ambientais, como; ondas de calor, enchentes, secas, tempestades severas e epidemias.

- Estimular a participação popular, para o engajamento das pessoas no investimento em energia limpa, combate ao uso de combustíveis fósseis e o abuso de agrotóxicos, estimulando a agricultura sustentável e familiar, desenvolvendo o potencial econômico da biodiversidade, fortalecendo as agências de proteção ambiental.

- Entre os principais documentos que apontam a degradação do planeta Terra, afetando os ecossistemas com a decorrente produção de doenças e agravamento das condições ambientais, com a promoção da extinção de espécies animais e vegetais, contaminação do solo, das águas e da atmosfera e redução da biodiversidade, colocando em risco a saúde e a própria sobrevivência futura da humanidade, destacam-se a Carta da Terra, a Declaração Universal sobre Bioética e Direitos Humanos da UNESCO e a Encíclica Laudato Si.

A degradação dos ecossistemas também vem acompanhada do agravamento das condições da vida humana e dos sistemas sociais, em total desacordo com o desenvolvimento científico que deveria determinar uma era de abundância, paz e felicidade. Entre as pessoas, a iniquidade e o aumento das desigualdades sociais são causas de morbimortalidades e desajustes que afetam a saúde planetária.

A prioridade do desenvolvimento econômico deve ser substituída pela prioridade à vida. Buscar o desenvolvimento sustentável a serviço de uma vida sustentável, limitando a obstinação por lucro e poder.

Por fim, está claro que os perigos que enfrentamos exigirão ações coletivas de conscientização no campo econômico, político e social. A cooperação universal é indispensável para a nossa sobrevivência.

Convidados: Carlos Dora, Carlos Nobre e Enrique Barros

Promotor: Grupo Hospitalar Conceição S/A

Apoiadores: Wonca, UFCSPA, UFRGS e UNISINOS. 


\section{Conflito de interesses}

Declaram não haver.

\section{Referências}

1. Boyden S. The Biology of Civilisation: Understanding Human Culture as a Force in Nature. Sydney: University of New South Wales Press; 2004.

2. Whitmee S, Haines A, Beyrer C, Boltz F, Capon AG, de Souza Dias BF, et al. Safeguarding human health in the Anthropocene epoch: report of The Rockefeller Foundation-Lancet Commission on planetary health. Lancet. 2015;386(10007):1973-2028.

3. Organização das Nações Unidas [Internet].Transformando Nosso Mundo: A Agenda 2030 para o Desenvolvimento Sustentável 2015 [acesso 2017 Dez 29]. Disponível em: https://nacoesunidas.org/pos2015/agenda2030/ 